\title{
Depleted diffuse bands in circumstellar envelopes of post-AGB stars
}

\author{
N. L. J. Cox ${ }^{1}$, R. Luna ${ }^{2}$, M.A. Satorre ${ }^{2}$, D.A. García Hernández ${ }^{3}$, \\ O. Suárez ${ }^{4}$, and P. García Lario ${ }^{1}$ \\ ${ }^{1}$ Herschel Science Centre, European Space Astronomy Centre, European Space Agency, \\ Villanueva de Cañada, Madrid, Spain; email: nick.cox@sciops.esa.int \\ ${ }^{2}$ Laboratorio de Astrofísica Experimental, Escuela Politécnica Superior de Alcoy, Universidad \\ Politécnica de Valencia, Alcoy, Alicante, Spain \\ ${ }^{3}$ W. J. McDonald Observatory, The University of Texas at Austin, Austin, Texas, USA \\ ${ }^{4}$ UAN, Université de Nice Sophia Antipolis, Nice, France
}

\begin{abstract}
We report on new results in the search for diffuse bands, signatures of still unknown origin, in the circumstellar envelopes of evolved (post-AGB) stars.
\end{abstract}

Keywords. Circumstellar matter, stars: AGB and post-AGB, ISM: lines and bands

\section{Diffuse interstellar bands}

The diffuse interstellar bands (DIBs) constitute a group of $\sim 300$ interstellar (IS) optical absorption features (Galazutdinov et al. 2000) observed ubiquitously throughout the Universe (see Cox \& Cordiner 2008). DIB strengths correlate roughly with the observed IS reddening by dust (e.g. Herbig 1995). Although not one of the DIBs has been identified there is strong evidence for a molecular organic nature of the carriers (Sarre 2008).

It is well known that evolved stars replenish the interstellar medium (ISM) during their mass loss phases. Depending on the chemistry, carbon-rich compounds can form in these outflows, which thus act as cosmic factories of complex organic matter. Despite unavoidable processing of such organic matter when exposed to the harsh UV conditions of the ISM one could foresee a direct connection with the carriers of the DIBs. Diffuse band (DB) carriers, or their pre-cursors, may be formed already in the circumstellar (CS) envelopes of evolved stars. In order to investigate this scenario we started a program to search for DBs in CS envelopes of post-AGB stars.

\section{Search for diffuse circumstellar bands}

Post-AGB stars are stars in a short-lived transition phase between AGB stars and protoplanetary nebulae. In this phase the remnant AGB shell is seen superimposed on the stellar spectrum (from early to late spectral type) of the central star. The lines-ofsight toward these targets usually probe both interstellar and CS matter. Therefore, it is important to derive the relative contribution of each component to the total observed reddening. Once the line-of-sight IS reddening is derived (e.g. with the extinction map by Drimmel et al. 2003) one can use the Galactic relationship between DIB strength and IS reddening to correct the observed DB strength and reddening to obtain the corrected DCB strength and CS reddening.

We divided our sample of 33 stars/sightlines in two groups; those dominated by CS and IS reddening, respectively. Figure 1 shows the $5780 \AA$ AB strength versus reddening. 


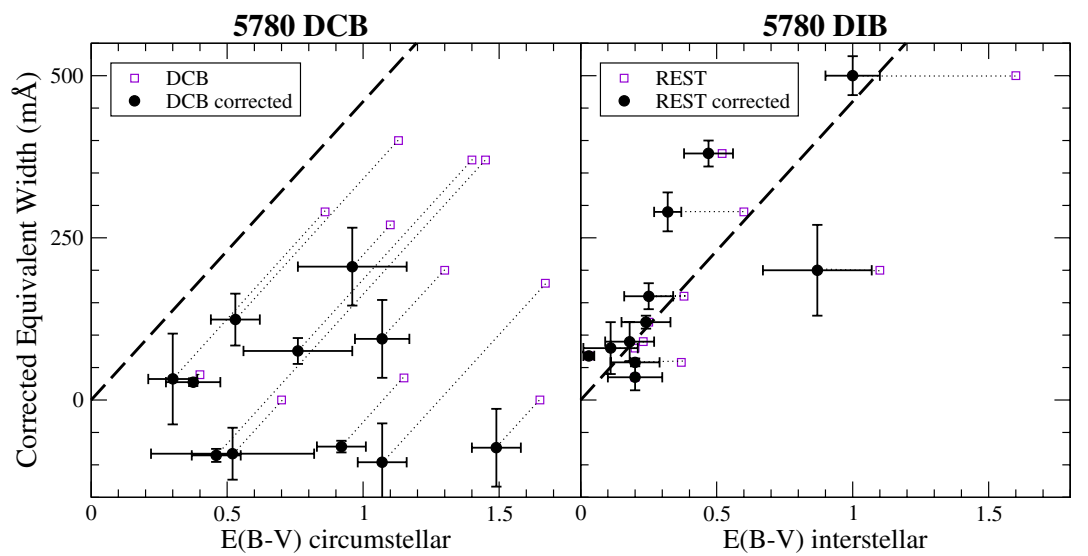

Figure 1. The corrected equivalent widths of the 5780 DCBs and DIBs versus the inferred CS and IS reddening, respectively. The thick dashed line represents the Galactic relationship: $\mathrm{EW}(5780) / E(B-V)=460 \mathrm{~m} \AA$. For the CS correction (right panel) we assume a total absence of DCBs: $\mathrm{EW}(5780) / E(B-V)=0$. Error bars are identical for uncorrected and corrected values. Uncertainties in the Galactic relationship are not considered.

Uncorrected equivalent widths and reddening are indicated by squares, while corrected equivalent widths (for IS and CS contribution in the left and right panel, resp.) and reddening are plotted as filled circles (with error bars).

\section{Results and future work}

For the IS group the observed DB strengths are in line with the Galactic DIB relationship with IS reddening. On the other hand, the inferred DCB strengths are systematically weaker for the inferred CS reddening, than expected from the IS trend. Note that positive values represent upper limits to the possible presence of DCBs since stellar line contamination has not been taken into account. A full description of the sample, data analysis and results, also for additonal DBs, is given in Luna et al. (2008).

Our work shows that (statistically) the carriers of IS DBs are not present - at least not in a detectable state - in CS envelopes of post-AGB stars. Processing of the organic CS matter by UV photons may be required to give rise to DIBs. Thus, although DIB carriers are not present in CS envelopes their pre-cursors could be. It would therefore be interesting to search for their absorption features in the UV/optical spectra of post-AGB stars. To detect these weak CS features we will need to accurately remove or separate the interstellar and atmospheric "contamination" from the CS spectrum. One possibility is to select targets with high radial velocities (such that IS and CS components have separate velocities) and whose line-of-sight extinction is dominated by the CS envelope.

\section{References}

Cox, N. L. J. \& Cordiner, M. A. 2008, this volume

Drimmel, R., Cabrera-Lavers, A., \& López-Corredoira, M. 2003, A\&A A, 409, 205

Galazutdinov, G. A., Musaev, F. A., Krelowski, J., \& Walker, G. A. H. 2000, PASP, 112, 648

Herbig, G. H. 1995, ARA\&A, 33, 19

Luna, R., Cox, N. L. J., Satorre, M., García-Hernández, A., Suárez. O., \& García-Lario, P. 2008, $A \mathscr{E} A, 480,133$

Sarre, P. J. 2008, this volume 